\title{
COSTES SOCIALES Y TEMPORALES DE LA MOVILIDAD DE LOS POLÍGONOS INDUSTRIALES EL CASO DE LA REGIÓN METROPOLITANA DE BARCELONA*
}

\author{
Àngel CebolladA ${ }^{1}$ \\ Pau G. Avellaneda ${ }^{1}$
}

\begin{abstract}
Resumen - Desde los años 60 del siglo XX se observa una tendencia creciente a trasladar las actividades industriales a polígonos creados para tal fin en las periferias urbanas. La conexión entre estos nuevos espacios productivos y el resto de espacios urbanos se ha fundamentado en el uso masivo del vehículo privado; esta opción de conectividad conlleva unos altos costes de movilidad los cuáles se dividen en operativos, ambientales, sociales y temporales. Este artículo se centra en el estudio de los costes sociales y temporales derivados de la movilidad a los polígonos industriales de dos municipios periféricos de la Región Metropolitana de Barcelona. Los resultados obtenidos, permiten realzar la importancia de la organización territorial y de la planificación de los transportes en la disminución de dichos costes y, por lo tanto, como instrumentos útiles para llevar a cabo políticas sociales.
\end{abstract}

Palabras clave: Polígonos industriales, movilidad, accesibilidad, costes sociales, costes temporales, Región Metropolitana de Barcelona.

Resumo - CUSTOS SOCIAIS E TEMPORAIS DA MOBILIDADE NOS POLÍGONOS INDUStriais. O CASO DA REgião metropolitana de BARCElona. Desde os anos 60 do século XX que há tendência para transferir as actividades industriais para novas zonas de actividade económica, encravadas nas periferias urbanas. A conexão destes novos espaços produtivos com outros espaços urbanos liga-se ao uso massivo do automóvel privado; esta opção de conectividade acarreta importantes custos de mobilidade, que se podem dividir em operativos, ambientais, sociais e temporais. Este artigo centra-se no estudo dos custos sociais e temporais da mobilidade, relacionados com polígonos de dois municípios periféricos da Região Metropolitana de Barcelona. Os resultados obtidos realçam a importância da organização territorial e da planificação dos transportes na atenuação destes custos, os quais são instrumentos úteis na elaboração de políticas sociais.

Palavras-chave: Parques industriais, mobilidade, acessibilidade, custos sociais, custos temporais, Região Metropolitana de Barcelona.

* Recebido: 8/04/2007. Revisto: 2/12/2007. Aceite: 18/12/2007.

1 Profesores del Departamento de Geografía. Universidad Autònoma de Barcelona. España. E-mail: Angel.Cebollada@uab.cat e E-mail: Pau.Avellaneda@uab.cat 


\begin{abstract}
THE SOCIAL AND TEMPORAL COSTS OF MOBILITY IN INDUSTRIAL DISTRICTS. The CASE of THE BARCElona Metropolitan Region. Since the 1960s, there has been an increasing tendency towards relocating industrial activities to areas located in the urban peripheries that are destined for that specific purpose. The private car has become the main means of connecting these production spaces with the rest of the urban fabric. This connectivity option entails high mobility costs, which can be analytically divided into operative, environmental, social and temporal costs. This article focuses on both the social and the temporal costs of the journeys to and from industrial estates located in two peripheral municipalities of the Barcelona Metropolitan Region. The main findings of the research highlight the important role played by territorial organisation and transport planning in reducing these costs, as well as their consequent usefulness when adequately embedded into social policies.
\end{abstract}

Key words: Industrial estates, mobility, journey, accessibility, social cost, temporal cost, Barcelona Metropolitan Region.

Résumé - COUTS SOCIAUX ET TEMPORELS DU DEPLACEMENT DES POLYGONES INDUSTRIELS. LE CAS DE LA REGION METROPOLITAINE DE BARCELONE. On observe, depuis les années 60, une tendance croissante au déplacement des activités industrielles vers de nouvelles zones, situées à la périphérie des villes. La liaison entre ces nouveaux espaces productifs et le reste de l'espace urbain a été basée sur l'usage massif des véhicules privés. Cette option a impliqué des coûts de mobilité élevés, tant économiques qu'environnementaux, sociaux et temporels. La présente étude porte sur les coûts sociaux et temporels dérivés de la mobilité liée aux zones industrielles de deux municipalités périphériques de la Région Métropolitaine de Barcelone. Les résultats obtenus permettent de souligner l'importance qu'auraient l'organisation territoriale et la planification des transports pour réduire ces coûts et permettre d'élaborer une politique sociale.

Mots-clés: Zones industrielles, mobilité, accessibilité, coûts sociaux, coûts temporels, Région Métropolitaine de Barcelone.

\title{
I. INTRODUCCIÓN
}

Existen en la actualidad un gran número de polígonos industriales y de actividad económica en Cataluña; según el trabajo Anàlisi de l'oferta i la demanda de polígons industrials $i$ terciaris a Catalunya realizado por el Instituto de Estudios Regionales y Metropolitanos de Barcelona (Miralles y Donat, 2007), en el año 2006 se contabilizaban hasta 1750 polígonos de actividad con una superficie superior a la media hectárea en el conjunto del territorio catalán. En total suma 32.240 hectáreas de las que casi la mitad (el 48,5\%) se hallan en la Región Metropolitana de Barcelona.

Aunque la aparición de dichos polígonos data de finales de los años 50 del siglo XX, momento en que empezó a producirse la instalación de industrias fuera de la trama urbana tradicional, no es hasta algunas décadas después que su presencia se generaliza en el territorio (Capel, 2005). 
No existe una sola causa para explicar la creación generalizada de estos polígonos sino que es fruto de la convergencia de distintos motivos. Los más evidentes serían: los cambios acontecidos en el proceso productivo que lleva a la fragmentación y a la localización diferenciada en el territorio de cada una de las fases de producción y, en relación a este último hecho, el aumento de la subcontratación y por tanto la creación de una estructura dominada por pequeñas empresas y, una vez más, a la difusión territorial del proceso de producción; las disfunciones que las industrias crean en el interior de las ciudades como ruidos, polución atmosférica o congestión; los precios del suelo urbano que expulsan las actividades industriales de las áreas urbanas tradicionales; los principios funcionalistas de la Carta de Atenas que rige la actuación urbanística y según los cuáles cada función urbana debe ubicarse en un espacio concreto huyendo de la promiscuidad de usos (López de Lucio, 1995, Massey, 1990; Marques, 1992).

La aparición de estos polígonos ha supuesto el incremento de la distancia física entre el espacio de la esfera laboral y el de los espacios del resto de la vida cotidiana (residencia, ocio, compras, etc.) y, con ello, a la necesidad de utilizar medios de transporte motorizados ${ }^{2}$. Para solucionar este requerimiento de movilidad, las políticas públicas han priorizado el uso del vehículo privado de manera que en estas últimas décadas se ha asociado el acceso al lugar de trabajo con el automóvil. El transporte público y las otras formas de transporte colectivo han quedado relegados a un papel secundario cuando no directamente marginal: la mitad de los polígonos de la Región Metropolitana de Barcelona no cuentan con ningún tipo de parada o estación de transporte público a una distancia inferior de 1500 metros (PIRMB, 2003) y sólo el 10,3\% de ellos (y el 7,4\% de los de Cataluña) tienen al menos una empresa que ofrezca un servicio de transporte colectivo propio.

Este artículo tiene por objeto analizar los costes sociales y temporales derivados de la actual situación de accesibilidad a los polígonos industriales. Se parte de la hipótesis que la planificación de la movilidad a estas áreas productivas se ha realizado menospreciando, cuando no ignorando, las implicaciones sociales que en este artículo tratamos como costes. Para su estudio se ha utilizado la metodología cualitativa en base a entrevistas en profundidad como forma de análisis de la estructura abordada. Los resultados se basan en el estudio de caso realizado en los municipios Polinyà y Palau-solità i Plegamans en la Región Metropolitana de Barcelona.

\section{LOS COSTES SOCIALES Y TEMPORALES COMO PARTE DE LOS COSTES GLOBALES DE LA MOVILIDAD}

La distribución territorial de los polígonos industriales y la priorización del uso del vehículo privado como instrumento hegemónico para el acceso al lugar

2 Hasta entonces, el acceso a los trabajos industriales era básicamente a pie o en bicicleta tal como ponen de manifiesto Miralles y Oyón (1998) en un estudio para la ciudad de Barcelona. 
de trabajo supone la asunción individual, y también colectiva, de determinados costes. Desde el campo de estudios de la "economía ecológica del transporte" (Estevan y Sanz, 1996) se incluyen en los costes globales de la movilidad, además de los "internos" (en los que el enfoque económico neoclásico focaliza su atención), todos aquellos impactos no "deseables" de esta actividad que tradicionalmente eran considerados externalidades del sistema y que, en esta propuesta quedarían incluidos en la contabilidad de los costes al apoyarse en el marco conceptual de la "economía de sistemas abiertos" (Miralles, 2002). A pesar de las diferencias en la clasificación de dichos costes, se pueden distinguir cuatro grandes categorías (Miralles y Cebollada, 2003).

- Los costes operativos son los más evidentes por ser los más visibles. Se dividen en costes de operación y de infraestructura. Los primeros son aquellos vinculados a la operación directa del medio de transporte (combustible, neumáticos, reparaciones, etc.) pero también a una parte de la gestión indirecta (como la amortización del vehículo o el seguro) que el usuario debe cubrir. Por su lado, los segundos hacen referencia a la construcción, mantenimiento y vigilancia de las infraestructuras necesarias para la circulación de los distintos medios de transporte. Se trata de un coste que la colectividad suele pagar a través de impuestos.

- Los costes ambientales han centrado la atención de numerosos trabajos (Whitelegg, 1997; Newman y Kenworthy, 1999; Lenzen, et al., 2003; Holmén y Niemeier, 2003; Ambrosino et al., 2004) especialmente desde el advenimiento de la crisis ambiental y del cambio global (Lemkow, 2002). Se relacionan especialmente con el impacto en la base ecológica del planeta (agotando sus recursos) y con la capacidad de carga del mismo por las emisiones de gases contaminantes (Maddison et al., 1996). Estos costes pueden resumirse en el consumo energético, la contaminación atmosférica y acústica, la fragmentación del territorio y su ocupación por la construcción de infraestructuras.

- Los costes sociales son diversos y afectan a diferentes colectivos sociales. Por un lado, y en relación directa con los ambientales, se encuentra la incidencia de la contaminación ambiental causada por el transporte en la salud pública. Este tema ha sido el objeto central de estudio de autores e instituciones públicas preocupados por las afecciones sobre patologías humanas de dicha contaminación; así se ha estudiado la población expuesta a niveles sonoros superiores a los recomendados por la UE (EEA, 2001; García y Garrido, 2003); los ingresos hospitalarios relacionados con aumentos de contaminación atmosférica o la sobremortalidad causada por esta contaminación (Moncada, 2001). Asimismo, los accidentes causados por los medios de transporte por carretera son otra afectación importante sobre la salud pública. En este sentido, estos accidentes son una de las primeras causas de muerte en las sociedades occidentales y la primera cuando se trata de muertes violentas; sólo en la 
Unión Europea se producen 113 muertos diarios en accidentes de tráfico (Campbell, 2007). También tienen una importancia creciente en el mundo del trabajo; en España, por ejemplo, una de las primeras causas de muerte en accidente laboral son los accidentes "in itinere"3, o sea, aquellos desplazamientos que se realizan para ir a, o volver desde, el lugar de trabajo (CONC, 2004): en concreto, en el año 2006 fallecieron 372 personas en estos accidentes y otras 1974 resultaron gravemente heridas (Idescat, 2007). Asimismo, en los costes sociales deben incluirse la expulsión de usos y usuarios del espacio público que puede sintetizarse en la pérdida de espacio público como lugar de relación y socialización y la pérdida de autonomía de movilidad de las personas mayores o los niños (Ryeng, 2002). Finalmente, cabe mencionar la exclusión social derivada de la incapacidad de desplazarse por no disponer de un vehículo propio y por la falta de oferta de transporte publico. Este hecho supone la imposibilidad de acceder a bienes y recursos (Avellaneda, 2007; Lucas, Grosvenor y Simpson, 2001; Preston y Rajé, 2006; TRaC, 2000; SEU, 2003) y, especialmente, a lugares de trabajo (Cebollada, 2007a; Cervero et al. 2002; Raphael y Rice, 2002; Sanchez, 2002).

- Los costes temporales también se incluyen en la cesta de afectaciones de la necesidad de movilidad. Si se considera que los desplazamientos son una actividad derivada, o sea, que se ejecuta para realizar otro quehacer (trabajar, comprar, realizar gestiones, etc.) y no tiene una finalidad en si misma, el tiempo deviene una variable a minimizar. Hay diferentes propuestas para contabilizar el tiempo invertido en un desplazamiento, desde aquél que se restringe exclusivamente al uso de un vehículo hasta el "tiempo social" destinado a la generación de desplazamiento (Illich, 1974; Naredo y Sánchez, 1992). La fórmula más utilizada para contabilizar el tiempo invertido en un desplazamiento es el transcurrido de "puerta a puerta", desde que se sale del origen hasta que se llega al destino final y puede empezar a realizarse la actividad propiamente dicha (Miralles, 2002). En uno de los pocos trabajos sobre la evolución del tiempo invertido para ir al trabajo en un arco temporal largo, Pooley y Turnbull (1999) afirman que desde 1890 hasta 1998 el periodo de trayecto en la Gran Bretaña se ha doblado pasando de una media de 17,7 minutos a finales del siglo XIX hasta los 34,5 minutos a finales de siglo $\mathrm{XX}$.

Es evidente que estos costes se relacionan de una manera muy directa con la distribución de las actividades urbanas en el territorio (Miralles y Cebollada, 2003): así, aquella organización territorial que minimice la movilidad y la nece-

3 En España los accidentes de ida o regreso al trabajo (in itinere) son considerados accidentes laborales. 
sidad de largos recorridos presentará unos costes menores que aquél territorio que tienda a separar usos y a crear grandes distancias entre las diferentes funciones urbanas.

Estos costes, evidentemente, también variarán según que medios de transporte sean los implantados en este territorio. Diferentes autores (Naredo y Sánchez, 1992; Noy, 1998; Requena, 2001) coinciden en que estos son más elevados en el caso del vehículo privado y ponen de manifiesto que la opción de priorizar un modelo de movilidad basado en el automóvil mientras las formas de transporte colectivo quedan relegadas a un papel conector secundario es la apuesta más costosa, tanto en términos individuales como colectivos. Un informe realizado por la ATM (2000) sobre los costes globales del transporte de viajeros en la Región Metropolitana de Barcelona reafirmó que los costes más elevados correspondían al coche, 0,84 euros/viajero-km, mientras que los de los transporte público apenas alcanzaban 0,51 euros/viajero-km.

En los resultados del trabajo de campo que se presentan en la parte final de este artículo se analizan dos de los costes que componen la contabilidad total de la movilidad: los sociales y los temporales los cuáles son los más ocultos y los más óptimos de abordarse mediante metodologías de estudio cualitativas.

\section{III. ÁREA DE ESTUDIO Y METODOLOGÍA}

Polinyà y Palau-solità ${ }^{4}$ son dos municipios adyacentes con una fuerte especialización industrial que pertenecen a la comarca del Vallès Occidental, en la provincia de Barcelona (fig. 1). Como otras poblaciones de la periferia metropolitana, las últimas décadas se han caracterizado por el incremento demográfico y, sobre todo, por la localización en ellos de actividades industriales. Esta dinámica ha generado un paisaje urbano donde se combina la presencia de nuevas áreas residenciales de baja densidad junto a extensas superficies industriales: en Polinyà los polígonos de actividad ocupan un 76,5\% de la superficie urbanizada municipal (201,07 Ha en total), mientras que en Palau-solità esta cifra desciende hasta el 48,2\% (403,212 Ha de suelo urbano total). El peso de la industria en ambos municipios se refleja en los lugares de trabajo localizados (LTL) en cada municipio de este sector de actividad en relación a la población ocupada residente (POR) en la industria: en Polinyà la relación entre ambos indicadores es de 3,02 (7281 LTL frente a 2409 POR) mientras que en Palau-solità es de 1,60 (9120 LTL frente a 5701 POR).

4 El nombre completo de este municipio es Palau-solità i Plegamans pero para facilitar la lectura en este artículo utilizaremos sólo la denominación de uno de los núcleos históricos del término. 


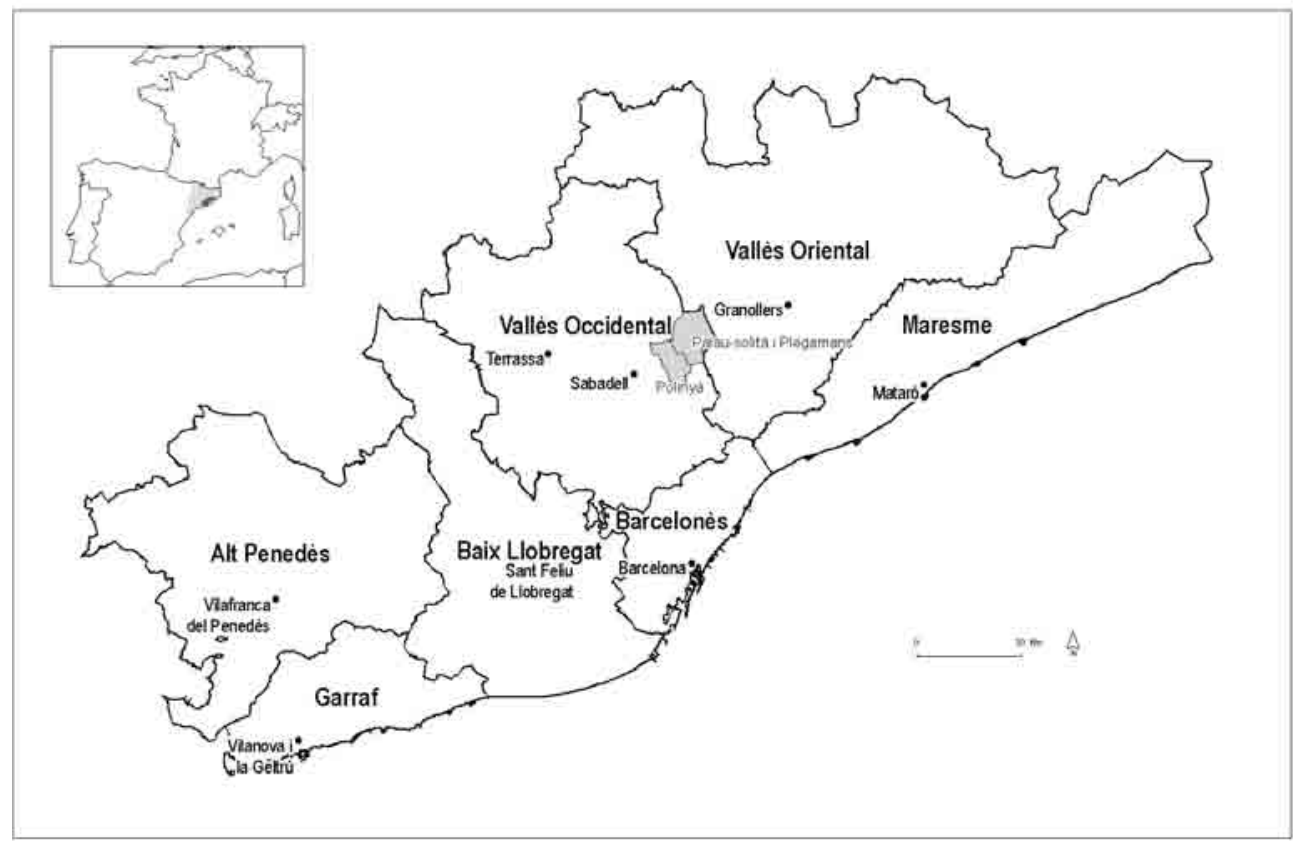

Fig. 1. El área de estudio en la Región Metropolitana de Barcelona Fig 1. Location of the study area in the Barcelona Metropolitan Region

La superficie industrial de los dos municipios se reparte entre siete polígonos, tres en Polinyà y cuatro en Palau-solità, formando dos grandes paquetes industriales al sur de los núcleos residenciales y a lo largo de las principales vías de comunicación (B-142 y C-59) que se completan con manchas menores de superficie industrial en el sector norte (fig. 2).

El número de empresas allí ubicadas era de 641 según datos del Pacto Industrial de la Región Metropolitana de Barcelona ${ }^{5}$ para el año 2004. En Polinyà domina una estructura industrial de pequeñas empresas que se refleja en un mayor número (377) a pesar de contar con un superficie de polígonos menor respeto Palau-solità que cuenta con 264 empresas. Este último municipio se caracteriza por la presencia de empresas multinacionales de dimensiones mayores, tanto en superficie como en trabajadores.

A pesar de las importantes necesidades de movilidad ${ }^{6}$ que estos polígonos generan, la oferta de transporte público ha sido menospreciada y es insuficiente

5 El Pacto Industrial de la Región Metropolitana de Barcelona es una asociación que incluye a las administraciones públicas (gobierno catalán y gobiernos locales), agentes sociales y universidades.

6 En este artículo sólo se aborda la movilidad cotidiana de personas y obviamos el transporte de mercancías. 


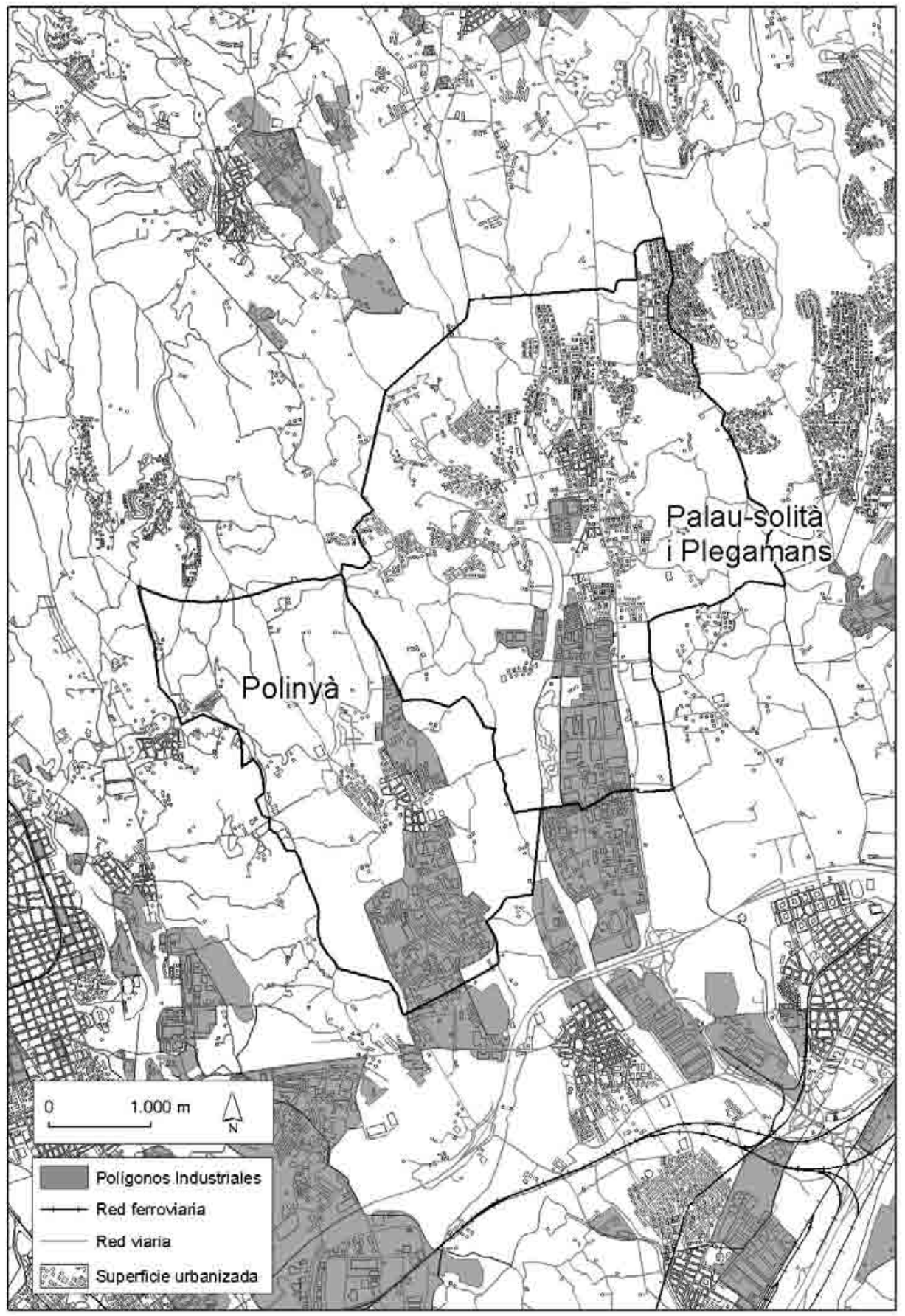

Fig. 2. Los polígonos industriales de Palau-solità y Polinyà Fig. 2. The industrial estates of Palau-solità and Polinyà 
para responder a la demanda actual. De hecho, la pobre oferta existente esta dimensionada para unas características que desaparecieron durante el último cuarto de siglo XX, cuando estos municipios presentaban unos altos índices de autocontención, unas dimensiones demográficas mucho más reducidas que las actuales, un marcado carácter rural y todavía no se habían integrado a las dinámicas metropolitanas (Anguera y Casas, 1990). En este contexto, las pocas expediciones de autobuses que dan servicio priorizan la cobertura de las áreas residenciales (igualmente insuficientes por las dinámicas de movilidad de principios de siglo XXI) mientras que las áreas de actividad han quedado hasta ahora al margen de las prioridades de la planificación de transporte público. Un reflejo de esta oferta inadecuada es su cuota de utilización: un $0,75 \%$ del personal trabajador de los polígonos de Polinyà y el 3,75\% de los de Palau-solità. Otro medio de transporte que cada vez juega un papel más residual en la conexión de los polígonos industriales es el autocar de empresa: sólo el 1,75\% de los trabajadores de Polinyà y el $1,0 \%$ de los de Palau-solità lo utilizan para ir al trabajo. La oferta de este último medio sufre un retroceso paulatino; en los inicios de los polígonos, especialmente en Polinyà, algunas de las primeras empresas ofrecían este servicio a sus trabajadores. Pero el aumento de la motorización y la absoluta priorización del coche privado han reducido su existencia a unas pocas empresas. Además, el derecho de uso no suele extenderse al conjunto del personal contratado puesto que, en la actualidad, se trata de un derecho adquirido por una parte de los trabajadores (los más antiguos) pero no por su totalidad (los más "nuevos" o aquellos que han renunciado a él). En estos momentos, este medio de transporte está percibido por parte de las empresas como una rémora del pasado que lo mantienen a regañadientes a causa de los acuerdos que en su día suscribieron con los organismos de representación de la plantilla. El resultado de todo ello es que el acceso a los polígonos debe realizarse en vehículo privado. Así, en el conjunto de polígonos de Polinyà, el 95,02\% del personal trabajador accede a su puesto de trabajo en coche (el 85,04\% como conductor y el 9,98\% como acompañante); para los polígonos de Palau-solità la cuota de uso del automóvil se sitúa en un $93 \%(85,0 \%$ como conductor y el $8,0 \%$ como acompañante).

\section{Metodología}

En este artículo se presentan los resultados obtenidos en una investigación llevada a cabo con métodos cualitativos. El carácter del trabajo, que tenía que enfrentarse a un universo difuso de estudio para identificar a los colectivos presentes y potenciales de los polígonos, conocer las estrategias de movilidad utilizadas para acceder a los lugares de trabajo y descifrar las percepciones asociadas a dichas estrategias, determinaron el uso de esta metodología de investigación. No obstante deben tenerse claro de antemano ciertas lagunas que desde la metodología cualitativa no podrán cubrirse; si bien permite hacer aflorar ciertos temas y conocer la estructura profunda de la problemática estudiada, 
estos métodos de análisis no permiten hacer una cuantificación del objeto de análisis. De todos modos, ambas metodologías (cuantitativa y cualitativa) lejos de ser contrapuestas pueden complementarse mutuamente identificando y definiendo un objeto para cuantificarlo posteriormente.

Así, se ha utilizado como herramienta de recogida de información la entrevista en profundidad, un elemento habitual en la investigación cualitativa. Estas entrevistas no son dirigidas, están realizadas a partir de la existencia de un guión abierto, lo que posibilita acceder a información que incluso no se preveía en un principio y permite al investigador incidir en ellas con una mayor profundidad si lo ve conveniente. Todas las entrevistas se grabaron en audio y se transcribieron de manera literal para ser analizadas. El análisis se basó en los procedimientos característicos de la "grounded theory" (Strauss y Corbin, 1997).

En total se realizaron treinta entrevistas en profundidad, repartidas teniendo en cuenta los agentes participantes en los polígonos industriales. El trabajo de campo se inició entrevistando a las empresas y a la administración pública local a partir de la información facilitada por el Pacto Industrial de la Región Metropolitana de Barcelona y de los propios ayuntamientos. Las entrevistas realizadas con estos agentes sirvieron, entre otras cosas, para detectar otros agentes con problemáticas de movilidad específicas presentes en los polígonos como son institutos de educación secundaria, empresas de trabajo temporal y empresas de servicios. En la fase final del trabajo de campo se ha procedido a entrevistar a trabajadores de las industrias de los polígonos de los dos municipios para completar la información obtenida previamente.

\section{RESULTADOS}

En este apartado se presentan los principales costes sociales y temporales asociados a la localización suburbana de los polígonos de los municipios de Polinyà y Palau-solità y a su deficiente conectividad con medios de transporte alternativos al vehículo privado. A pesar de las conexiones entre las distintas esferas de los costes sociales analizados, aquí se presentarán según los colectivos o instituciones afectados siguiendo el modelo de Cebollada (2007b). Por ello, pueden distinguirse los costes que afectan a las personas trabajadoras que desarrollan su actividad laboral remunerada en los polígonos estudiados; los costes derivados de la exclusión laboral de ciertos colectivos; por último, las consecuencias que tienen dichos costes en la competitividad de las empresas que, de una forma u otra, operan en estos polígonos.

\section{Costes sobre la plantilla}

Para las personas que desarrollan su actividad profesional en alguno de los polígonos de Polinyà o Palau-solità, los déficits de accesibilidad en transportes colectivos pueden suponer una afectación sobre su salud. A pesar de que los 
diferentes agentes entrevistados no perciben los accidentes "in itinere" como un problema grave, esta incidencia ha sucedido en la práctica totalidad de las empresas entrevistadas (tanto a directivos como a trabajadores).

"R: Si, sé que ha habido algunos, no muchos, pero alguno sí que ha habido. Hombre muchos, muchos, no. El último año ¿cuántos pueden haber habido, uno o dos? Un par (...) Esto una gran incidencia no tiene.” (Trabajador 1)

Estos accidentes tienen un abanico de consecuencias muy amplias sobre la salud de la persona afectada que van desde la nula afectación física hasta la defunción del trabajador. Estos accidentes de tráfico se perciben como un tema genérico que pasa a la red vial en abstracto, pero que en numerosas ocasiones acontece en el mismo polígono, debido a la congestión de sus calles y carreteras en las horas punta de entrada y salida del trabajo.

"En Polinyà, a ver, de mis trabajadores yo creo que no pasa un año en el que no haya habido alguien que haya tenido un accidente, en un cruce concreto de la carretera de Sentmenat." (Empresa 5)

Asimismo, el tiempo invertido para poder llegar hasta el puesto de trabajo y las características urbanas de los propios polígonos dificultan la conciliación de la vida laboral y personal. Por un lado la congestión de la red viaria suponen un sobrecoste temporal que el individuo debe restar de su tiempo personal; en este sentido, la jornada laboral se alarga mucho más que los horarios de trabajo propiamente dicho ${ }^{7}$. Por otro lado, la monofuncionalidad propia de los polígonos dificulta dicha conciliación al no existir en este entorno urbano ningún tipo de servicio; este hecho impide realizar actividades personales tales como compras, gestiones u ocio en el mismo polígono. La monofuncionalidad es especialmente percibida como un elemento negativo por las personas que trabajan en oficinas que preferentemente hacen horario partido y mayoritariamente son mujeres.

“... pero en las oficinas si que tenemos gente a la que le hace gracia o la comodidad de poder acceder a unos buenos comercios o hacer una gestión en correos, es que todo es complicado..” (Empresa 3)

7 La congestión, en 2001 y para el conjunto de Catalunya, supuso una pérdida económica de tiempo de unos 323M de euros (DPTOP, 2001). En el caso del área de estudio los costes percibidos por los usuarios son superiores al cálculo "objetivo" (a partir de la distancia y una velocidad media de $50 \mathrm{~km} / \mathrm{h}$ ) de forma que demuestra el efecto de la congestión en el periodo de entrada al polígono (PIRMB, 2005). 


\section{Los colectivos con dificultades de acceso a los puestos de trabajo}

La ya mencionada localización de los polígonos de Polinyà y Palau-solità, la ineficiencia de los sistemas de transporte público y la práctica ausencia de transporte colectivo de empresa tienen un coste en forma de exclusión laboral para aquellos grupos que no disponen de un vehículo propio para acceder al lugar de trabajo aunque residan en su entorno territorial.

Los resultados de los análisis de las entrevistas ponen de manifiesto la existencia de un consenso generalizado entre los agentes de los polígonos de que la responsabilidad del desplazamiento de las plantillas hasta el lugar de trabajo debe recaer en los propios trabajadores. Ante este hecho, se establecen filtros que dificultan la contratación de aquellos individuos desmotorizados. Son numerosas las empresas que exigen que los solicitantes de empleo dispongan de vehículo propio para acceder al puesto de trabajo; en otros casos, sin embargo, esta exigencia no es explícita por que ya se "sobreentiende" o en todo caso cada persona puede asumir las estrategias de movilidad que crea convenientes para llegar a la firma.

No obstante, esta exclusión laboral se modula en función de las horas del día en que se quiera acceder a los polígonos y de la localización concreta de cada empresa. En referencia al primer caso, las posibilidades de acceso al lugar de trabajo en horario nocturno es absolutamente imposible en transporte público mientras que en la franja central del día existe una mínima oferta. Pero además de las diferentes accesibilidades horarias, la ubicación de la firma en el polígono es otro elemento que interviene en el nivel de exclusión: la distancia entre la parada del autobús y la puerta de la empresa y las condiciones del itinerario son los factores primordiales.

Dichas dificultades de acceso a los polígonos de Polinyà y Palau-solità se encuentran heterogéneamente repartidas en el seno de la estructura social y pueden distinguirse tres colectivos sociales con mayores cotas de exclusión: mujeres, jóvenes e inmigrantes.

Las mujeres más afectadas por la exclusión laboral relacionada con los déficits de accesibilidad son aquellas que presentan unos niveles más bajo de formación y que desarrollan su trabajo en producción o bien en los servicios externos como la limpieza. Para superar esta situación, las mujeres afectadas construyen estrategias de movilidad dependientes de terceras personas, lo que las convierte en usuarias del vehículo privado como acompañante. Asimismo, las posibilidades de acceso a los polígonos queda relegado a un ámbito territorial mucho más limitado que en el caso de las personas que disponen de vehículo privado. Por lo que hace a los polígonos de Polinyà, su radio de afluencia se limita al propio municipio siendo muy difícil el acceso desde otros municipios cercanos como Sabadell, centro del sistema urbano donde se inserta Polinyà. Los polígonos de Palau-solità también presentan un ámbito de afluencia local aunque también acceden mujeres sin coche, previo establecimiento de estrategias dependientes, provinentes del municipio vecino de Mollet del Vallès. De todas 
formas, dichas estrategias dependientes no son garantía de continuidad puesto que cualquier incidencia hace el puesto de trabajo inalcanzable.

“..Es más, la última que se iba con el de cámaras cuando el de cámaras plegó pues ella a los dos días cogió la baja, hasta que la echaron. Porque no podía ir para su casa, no tenía a nadie que la bajara.." (Trabajador 3)

Los jóvenes son otro de los colectivos con importantes déficits de accesibilidad a los polígonos de Polinyà y Palau-solità. Pero bajo la denominación "jóvenes" se encuentran dos grupos completamente distintos. Por ello es preciso distinguir a jóvenes trabajadores de los estudiantes de educación secundaria. El primero de los grupos lo constituyen individuos de edades bajas, sin formación y con pocos recursos económicos. Se trata de uno de los colectivos que presenta mayores dificultades de inserción laboral hecho que conduce a su desvaloración social y los lleva a situaciones de elevado riesgo de exclusión social severa.

"..A ver, normalmente la gente que no tiene transporte es gente joven que ha dejado los estudios y quiere empezar a trabajar y no sabe donde ir. Entonces, normalmente van a las ETTs a pedir trabajo." (ETT 1)

El segundo de los grupos de jóvenes lo forman los estudiantes que deben realizar prácticas profesionales de los ciclos formativos de la educación secundaria. Dentro de este grupo todavía puede distinguirse entre aquellos que cursan el ciclo formativo medio (con edades habitualmente inferiores a los 18 años) de los de ciclo superior, los cuáles ya son mayores de edad y, por tanto, presentan índices de motorización más elevados. Los que presentan mayores dificultades de accesibilidad territorial son los que siguen ciclos formativos medios y predominantemente hombres. Esta diferencia entre chicos y chicas se debe a los roles distintos de género que se asumen en el mundo laboral; mientras las mujeres optan preferentemente por unas profesiones (administración, sanidad, peluquería, comercio...) localizadas en su mayor parte en las tramas urbanas consolidadas, los hombres copan las ramas industriales por lo que los lugares de prácticas se concentran en gran medida en los polígonos. Las dificultades de estos estudiantes se agrandan debido a la necesidad de compatibilizar las prácticas profesionales en las empresas con la asistencia a las clases al instituto. Este hecho significa que la disponibilidad de tiempo para realizar el desplazamiento es corto, lo que les impide utilizar el transporte público por las escasas expediciones que realizan en los polígonos. Además, el uso del ciclomotor no se concibe como un instrumento apto para acceder a los polígonos de Polinyà y Palausolità por la percepción de la falta de seguridad vial: en el caso de Polinyà la peligrosidad se percibe en el interior de los polígonos mientras que en Palausolità esta falta de seguridad se localiza en su vía de acceso. 
El colectivo formado por los inmigrantes es el tercero nombrado anteriormente. Este colectivo presenta unas características particulares puesto que sus miembros son los que despliegan las estrategias de movilidad más variadas y extremas por los riesgos y sobrecostes asumidos. En este sentido, en muchas ocasiones su presencia en los puestos de trabajo de los polígonos responde a la asunción de unas pautas de movilidad que no serían asumidas por otros colectivos sociales. Así, el punto de inicio para acceder al empleo es mentir sobre la disponibilidad de vehículo privado, única forma posible para conseguir el trabajo. A partir de aquí deben utilizar distintas pautas de movilidad. Una de ellas es compartir el vehículo con otras personas, habitualmente de su mismo país de origen; para ello es preciso contar con una red de relaciones sociales fuerte. Pero en otras ocasiones los desplazamientos se hacen andando o en bicicleta, a pesar que se trata de itinerarios que transcurren por arcenes de carreteras, en horas extremas de la jornada y de varios quilómetros de distancia por lo que la caminata puede alargarse varias horas.

"Si no pueden pedir una moto a un amigo, van andando, tienen una bici. Ellos ya encuentran, ya." (ETT 1)

Otra estrategia utilizada por los inmigrantes es el uso del transporte público aunque ello les suponga, una vez más, largas caminatas hasta la parada, largas esperas a que pase el autobús y diferentes transbordos hasta llegar a destino.

\section{Las empresas y las condiciones de accesibilidad a los polígonos}

Para las empresas ubicadas en los polígonos de Polinyà y Palau-solità, la falta de una oferta de transporte colectivo adecuado a sus necesidades repercute en su competitividad. Es, en cierto modo, la vuelta de los costes expuestos anteriormente para la plantilla y para colectivos excluidos que finalmente afecta a las propias empresas. Posiblemente, una de las principales consecuencias es que estas firmas se ven en la necesidad de operar en un mercado laboral reducido, especialmente cuando afecta a las categorías profesionales extremas, principalmente las más bajas pero también, en menor medida las más altas. La satisfacción de las necesidades de contratación de perfiles laborales menos especializados sólo puede realizarse recurriendo al mercado laboral del propio municipio a causa de la baja disponibilidad de vehículo de los individuos que conforman los colectivos con estas características profesionales. Por este motivo, hay empresas que acaban contratando a personas que no responden al perfil exacto deseado a pesar de que en municipios próximos de Polinyà (como Sabadell o Terrassa) y Palau-solità (como Mollet del Vallès) hay bolsas de demanda de trabajo que se ajustan más a las ofertas de las empresas, de modo que su productividad será más baja de la deseada.

“..al menos a la hora de buscar a gente tendríamos al menos mucha más oferta, porque la gente no vería venir a trabajar a Polinyà como un 
problema, y entonces también un ahorro porque las empresas están pagando un coste que realmente el trabajo que está desarrollando aquella persona no lo vale, pues que o esto o nada. Y porque agravado con el tema que te comentaba que el nivel de preparación de Polinyà no es precisamente el más elevado del mundo.." (Empresa 2)

Otro de los perfiles que las firmas tienen problemas para contratar (o para retener) es de las categorías profesionales superiores. En estos casos, el empleado valora cada vez más elementos como el tiempo libre; el sobrecoste temporal hasta el trabajo o hasta el hogar debido a la congestión de la red viaria para aquellos residentes en municipios relativamente alejados como es el caso de Barcelona son elementos que desincentivan a trabajar en los polígonos de Polinyà y revalorizan el criterio de proximidad.

“..nosotros a veces, a veces no de hecho incrementando este coste para incentivar a que la gente quiera desplazarse a la fábrica, a las oficinas pero también es cierto que cuando más elevado es el sueldo la gente empieza a valorar más otras cosas que no sea el dinero, también el tiempo de ocio, etc., etc. y como ya sabéis pues por ejemplo en Barcelona hay unos atascos monumentales para salir y para entrar en las horas punta que es el horario que trabaja la gente de las oficinas precisamente y esto produce un problema a la hora de captar a gente que creemos que es la adecuada, nos limita bastante.." (Empresa 3)

Las dificultades de acceso con que se encuentran los estudiantes de prácticas mencionadas anteriormente también tienen un coste sobre las empresas. La posibilidad de incorporar estos estudiantes es, en general, bien valorado por parte de las firmas de los polígonos porque lo perciben como una posibilidad de probar futuros trabajadores que ya tienen una formación básica pero al mismo tiempo pueden acabarse de formarse según las necesidades específicas de la empresa. A pesar de ello, las dificultades de accesibilidad impiden que este recurso esté al alcance de las firmas de los polígonos de estudio.

"..sobre todo teníamos un buen contacto con un centro de Mollet que ahora ha dejado de firmar convenios y entonces cuando planteaba la posibilidad de colaborar, convenio aqui en nuestra compañía pues ya veíamos que no teníamos a nadie que los pudiera traer aquí y que tardarían más de una hora en venir y entonces, salían tenían también el tiempo justo de acabar de estudiar y venir hacia aqui o sea no podían pasarse una hora de camino sino llegarian prácticamente a la hora de cerrar nosotros.." (Empresa 6)

Estas dificultades de accesibilidad que se están describiendo, redundan en el hecho que las empresas de los polígonos de los dos municipios de estudio se 
enfrenten con problemas para estabilizar sus plantillas. Los costes que debe asumir el personal empleado para acceder diariamente a los puestos de trabajo provoca que se produzcan rotaciones en el personal que busca nuevos empleos en ubicaciones más accesibles. Para los polígonos de Polinyà y Palau-solità se ha detectado que las empresas más afectadas por este hecho son aquellas que quedan más alejadas de la oferta del transporte público y aquellas pertenecientes a los sectores de actividad con unos salarios más bajos.

“..cuando a las empresas de Palau, de Polinyà, sobre todo estas a las que no llegan tanto los transportes, la oferta yo creo que hay más rotaciones. También es improductivo para las empresas, porque las empresas que estén en el polígono - donde no llega el transporte, hay empresas que tienen más problema de personal. No nos lo dicen directamente que es por el transporte, pero nosotros lo notamos. Porque si evidentemente tiene coche o moto al menos llegan bien" (ETT 2)

\section{CONCLUSIONES}

El estudio de los polígonos de Polinyà y Palau-solità i Plegamans ha mostrado los importantes costes sociales y temporales que acarrea las actuales condiciones de accesibilidad. Los costes sociales y temporales, lejos de ser un impacto diluido en el conjunto de la sociedad, afectan de una forma clara y precisa a todos los colectivos (tanto los presentes como los potenciales) que conforman la "población" de los polígonos. También las empresas que desarrollan su actividad en estos espacios ven afectada su capacidad productiva y con ello su competitividad.

La incorporación de los costes sociales y temporales realza el papel de la organización territorial y de la planificación de los transportes como instrumentos de política social; las distintas opciones de modelos urbanos y movilidad tienen una incidencia radicalmente diferente en los costes sociales. Los espacios suburbanos y monofuncionales como los polígonos de los dos municipios de estudio favorecen la generación de desplazamientos de movilidad cotidiana de larga distancia y, con ello, fomentan el uso de los medios de transporte motorizados los cuáles, a su vez, presentan mayores costes sociales. No obstante, tal como se ha expuesto en el artículo, dichos costes sociales varían notablemente según las opciones de transporte que se implanten en el territorio: mientras los transportes colectivos minimizan los impactos negativos en la sociedad, la apuesta por el vehículo privado como instrumento conector aumenta los costes ya que favorece la exclusión laboral, incide negativamente en la salud del personal trabajador y, a causa del mismo colapso del sistema, dificulta la compaginación de la vida personal y laboral. Como acabamos de enunciar, estos efectos repercuten negativamente en la competitividad de las empresas. 
El estudio de estas repercusiones sociales derivadas de la ubicación de los polígonos industriales en el territorio así como de la opción del sistema de transporte debe formar parte de las diagnosis de los Planes de Movilidad a estos espacios productivos. Desde la aprobación de la Ley 9/2003 de la Movilidad por parte del Parlamento de Cataluña, existe en esta comunidad autónoma una cierta profusión de planes que tienen como objetivo la mejora de la accesibilidad. De todos modos, en su gran mayoría, estos planes no incorporan, ni en la diagnosis ni mucho menos en las acciones propuestas, los costes sociales. La definición de la disminución de los costes sociales como objetivo central de dichos planes significa que previamente deben conocerse la diversidad de situaciones y estrategias en relación a la movilidad de los diferentes colectivos para poder diseñar y desarrollar acciones para tal fin. Estas acciones, probablemente, deberán incluir actuaciones específicas según colectivos así como el fomento de foros de debate y concertación entre todos los agentes de los polígonos. Por ello, los estudios sobre los costes sociales y temporales de la movilidad a los polígonos suponen un primer paso para definir nuevas políticas sociales desde la planificación del territorio y los transportes.

\section{BIBLIOGRAFIA}

Ambrosino G, Nelson J, Romanazzo M (ed.) (2004) Demand Responsive Transport Services: Towards the Flexible Mobility Agency. ENEA, Rome.

Anguera J, Casas J B (1990) L'economia del Vallès Occidental. De la indústria als serveis. Caixa d'Estalvis de Catalunya, Barcelona.

ATM (2000) Els comptes del transport de viatgers a la Regió Metropolitana de Barcelona. Autoritat del Transport Metropolità, Barcelona.

Avellaneda P (2007) Movilidad, pobreza y exclusión social. Un estudio de caso en la ciudad de Lima. Tesis de Doctorado, Universidad Autònoma de Barcelona, Bellaterra.

Campbell G (2007) Panorama of transport, Eurostat, Luxembourg. [Consultado el 15 de octubre de 2007]. http://epp.eurostat.ec.europa.eu/cache/ITY_OFFPUB/KS-DA-07-001/EN/KS-DA-07001-EN.PDF

Capel H (2005) La morfología de las ciudades. II Aedes facere: técnica, cultura y clase social en la construcción de edificios. Ediciones del Serbal, Barcelona.

Cebollada A (2007a) Aproximación a los procesos de exclusión social a partir de la relación entre el territorio y la movilidad cotidiana. Documents d'Anàlisi Geogràfica, 48:105-121.

Cebollada A (2007b) L'acessibilitat als polígons Industrials: una asignatura pendent. Papers Regió Metropolitana de Barcelona (Polígons d'activitat econòmica: tendències de localització $i$ accessibilitat), 45: 38-47.

Cervero R, et al. (2002) Transportation as a Stimulus of Wefare-to-Work. Private versus Public Mobility. Journal of Planning Education and Research, 200: 50-63.

CONC (2004) L'accés sostenible al lloc de treball. CCOO, Barcelona.

DPTOP (2001) Estudi dels costos socials i ambientals del transport a Catalunya. Generalitat de Catalunya, Barcelona. 
EEA (2001) TERM 2001. Indicators tracking transport and environment integration in the European Union. European Environment Agency, Copenhagen.

Estevan A, Sanz A (1996) Hacia la reconversión ecológica del transporte en España. Los Libros de la Catarata, Madrid.

García B, Garrido F (2003) La contaminación acústica en nuestras ciudades. Fundació la Caixa, Barcelona.

Holmén B, Niemeier D (2003) Air quality. In Hensher D, Button J (ed.) Handbook of Transport and the Environment. Elsevier, Oxford: 61-79.

IDESCAT (2007) Dades demogràfiques i de qualitat de vida. Institut d'Estadística de Catalunya, Barcelona. [Consultado el 18 de octubre de 2007]. http://www.idescat.net/dequavi/

Illich I (1974) Energía y equidad. Barral, Barcelona.

Lemkow L (2002) Sociología ambiental. Icaria, Barcelona.

Lenzen M, et al. (2003) Climate Change. In Hensher D, Button J (ed.) Handbook of Transport and the Environment. Elsevier, Oxford: 37-60.

López de Lucio R (1993) Ciudad y urbanismo a finales del siglo XX. Servei de Publicacions de la Universitat de València, València.

Lucas K, et al. (2001) Transport, the environment and the social exclusion. Joseph Rowntree Foundation, Layerthorpe.

Maddison D, et al. (1996) The True Costs of Road Transport. CSERGE, London.

Marques M (1992) Subcontratação e Autonomia empresarial: Um estudo sobre o Caso Português. Afrontamento, Porto.

Massey D (1990) Spatial Division of Labour. Social Structures and the Geography of Production. Macmillan, London.

Merlin P (1991) Geographie, economie et planification des transports. PUF, Paris.

Miralles C (2002) Ciudad y transporte. El binomio imperfecto. Ariel, Barcelona.

Miralles C (1997) Ciutat i transport. Reflexió sobre la Barcelona contemporània. Servei de publicacions de la UAB, Bellaterra.

Miralles C, Cebollada A (2003) Movilidad y transporte. Opciones políticas para la ciudad. Fundación Alternativas, Madrid.

Miralles C, Donat C (2007) Anàlisi de l'oferta i la demanda de polígons d'activitat a Catalunya. Papers, Regió Metropolitana de Barcelona, 45: 8-36.

Miralles C, Oyón J (1998) De la casa a la fábrica. Movilidad obrera y transporte en la Barcelona de entreguerras. In Oyón J (ed.) Vida obrera en la Barcelona de entreguerras. Centre de Cultura Contemporània de Barcelona, Barcelona: 159-201.

Moncada S (2001) Transport i salut laboral. Quaderns de l'Escola (Mobilitat, transport public $i$ treball), 4: 111-116.

Naredo J, Sánchez L (1992) Las cuentas del automóvil desde el punto de vista del usuario. Economía y Sociedad, 6:39-52.

Newbery D (1998) Fair Payment from road users: a review of the evidence on social and environmental. AA Policy Paper. The Automobile Association, Basingstoke.

Newman P, Kenworthy J (1999) Sustainability and cities. Overcoming automobile dependence. Island Press, Washington, D.C.

Noy P (1998) Una estimació dels costos reals de l'automòbil. Medi Ambient, 15:8-17. 
PIRMB (2005) Proposta de serveis per accedir als polígons industrials de la RMB. Pla de serveis: Polígons Polinyà i Palau-solità i Plegamans. PIRMB i DPTOP, Barcelona.

PIRMB (2003) Transport públic i treball. Beta editorial, Barcelona.

Pooley C G, Turnbull J (1999) The journey to work: a Century of change. Area, 31(3): 281-292.

Preston J, Rajé F (2007) Accessibility, mobility and transport-related social exclusion. Journal of Transport Geography, 15(3): 151-160.

Raphael S, Rice L (2002) Car ownership, employment, and earnings. Journal of Urban Economics, 52 (1): 109-130.

Requena R (2001) La comptabilitat del transport i el model de mobilitat en la relació entre Sabadell i la Universitat Autònoma de Barcelona. Disertación de Maestrado, Universitat Autònoma de Barcelona, Bellaterra.

Ryeng E (2002) Children in traffic - mode choice, motor skills, freedom and restrictions. In WALK $213^{\text {rd }}$ International Conference: Steps towards liveable cities. WALK 21, San Sebastián. [Consultado en 20 de septiembre de 2007] http:/www.walk21.com/papers/San\%20Sebastian $\% 2002 \% 20$ Ryeng\%20Children\%20in\%20Traffic.pdf.

Sanchez T (2002) The impact of public transport on US metropolitan wage inequality. Urban Studies, 39 (3): 423-436.

SEU (2003) Connections; final report on transport and social exclusion. Social exclusion unit. Ofifice of the Deputy Prime Minister, London.

Strauss A, Corbin J (eds) (1997) Grounded theory in practice. Sage, Thousands Oaks.

$\mathrm{TRaC}$ (2000) Social exclusion and the provision and availability of public transport. DETR, London.

Whitelegg J (1997) Critical mass: transport, environment and society in the twenty first century. Pluto, London. 